Journal of Economics, Finance and Accounting Studies (JEFAS)

ISSN: 2709-0809

DOI: $10.32996 / \mathrm{ijllt}$

Journal Homepage: www.al-kindipublisher.com/index.php/jefas/index

\title{
Impact de l'aide publique sur la croissance économique et la réduction de la pauvreté en République Démocratique du Congo
}

\author{
Murhula Balasha Benjamin ${ }^{1}$, Kitsali Katungo Jean-Hélène ${ }^{2}$ \& Mushagalusa Balasha Arsène ${ }^{3}$ \\ ${ }^{12}$ Faculté des Sciences Economiques et de Gestion, Université de Lubumbashi, PO Box 1825, Lubumbashi, République \\ démocratique du Congo \\ ${ }^{3}$ Unité d’Economie et Développement Agricoles, Université de Lubumbashi, PO Box 1825, Lubumbashi, République \\ démocratique du Congo \\ Corresponding Author: Murhula Balasha Benjamin E-mail: murhulabenjamin@gmail.com
}

ARTICLE INFORMATION

Received: November 21, 2020

Accepted: January 01, 2021

Volume: 3

Issue: 1

DOI: 10.32996/jefas.2021.3.1.1

\section{KEYWORDS}

Aide Publique au Développement, Croissance Economique, Pauvreté, RD Congo

\section{ABSTRACT}

La République démocratique du Congo figure parmi les plus grands pays bénéficiaires de l'aide publique au développement (APD) au monde et est comptée simultanément parmi les pays les plus pauvres du globe avec l'indice de développement humain et le revenu national brut par habitant les plus bas. Cela soulève de sérieuses questions sur l'efficacité de I'APD à promouvoir la croissance économique et à réduire la pauvreté dans le pays. Pour ce faire, les données relatives à l'APD de près de trois décennies (1990 à 2018) ont été triées de bases de données de la Banque Mondiale et de la Banque Centrale du Congo et ces dernières ont été soumises à une analyse économétrique par la méthode de moindre carré ordinaire pour comprendre l'impact de I'APD sur la croissance économique et la réduction de la pauvreté en RD Congo. Les résultats ont montré qu'après l'an 2000, l'aide publique a connu une reprise fulgurante après une tendance de vache maigre (1990-2000) et celle-ci a contribué à la croissance économique et à la réduction de la pauvreté. Cependant, l'effet de l'aide sur le niveau de pauvreté ne passe pas par le canal de croissance économique. La croissance ne contribue pas à la réduction de la pauvreté en RD Congo. En réalité, si la croissance peut se réaliser sans forcément entrainer la réduction de la pauvreté, ce qu'il s'agit d'un partage inégalitaire de richesses et/ou de la captation des fruits de la croissance par une élite au détriment du reste de la population. Ces résultats sont bel et bien un signal que le régime de croissance de l'économie congolaise reste fondamentalement non inclusif et masque de profondes disparités et de la misère au sein de la population. Les pistes d'amélioration de la gestion de l'aide publique sont proposées.

\section{Introduction}

Selon le Comité d'Aide au Développement (CAD) de l'OCDE, l'Aide Publique au Développement (APD) est l'ensemble des ressources fournies par les États industrialisés dans le but exprès de promouvoir le développement économique et d'améliorer les conditions de vie de la population dans les pays en développement. Pour que l'aide extérieure soit admise comme une APD, elle doit répondre aux caractéristiques suivantes : (i) provenir d'organismes publics y compris des Etats et des autorités locales ou de leurs agences d'exécution ou d'organismes agissant pour le compte d'organismes publics ; (ii) être acheminée vers les pays ou les territoires en développement ou à défaut à une institution multilatérale qui sera chargée d'acheminer cette aide vers de tels pays, en leur octroyant des prêts à des conditions très préférentielles ; (iii) avoir pour but essentiel de favoriser le développement économique et l'amélioration du niveau de vie des populations dans les pays

K C AL-KINDI CENTER $\mathbf{R}$ D FER RESEARCH Your gatewoy to world-class research
Published by Al-KindiCenter for Research and Development. Copyright (c) the author(s). This is an open access article under CC BY license (https://creativecommons.org/licenses/by/4.0/) 
bénéficiaires ; (iv) prendre enfin, la forme de dons, de prêts assortis de conditions de faveur (comportant un élément de libéralité d'au moins $25 \%$ du total) ou d'apports d'assistance technique. En effet, la République Démocratique du Congo(RDC) a depuis longtemps bénéficié de l'aide publique au développement de la part des partenaires multilatéraux et bilatéraux, qui l'accompagnent dans ses objectifs de croissance économiques et de réduction de la pauvreté. Entre 1960 et 1990, l'APD en direction de la RDC a connu un accroissement irrégulier certes mais réel. Elle est passée de 71,2 millions en 1960 à 715,3 millions USD en 1990 (Mandela R. et Naoutem B., 2009). Elle a doublé en 2002-2003 à cause de la reprise de la coopération structurelle avec les institutions internationales et elle a représenté en 2005, 40\% du PIB (PNUD, 2010) après une tendance baissière observée en 1990-1999 et a atteint un record historique de 5,526 milliards de dollars américains en 2011 (Banque Mondiale, 2018). Bien qu'elle ait connu une baisse depuis 2012, elle demeure très importante. Elle est estimée à plus de 2 milliards de dollars en 2018, soit près de 27 dollars d'aide par habitant. Entre 2010-2018, la RDC a bénéficié 8,5 milliards de dollars de donateurs multilatéraux dont les cinq grands donateurs ont été la Banque Mondiale, l'Union Européenne, le Fonds Mondial, la Banque Africaine de Développement et les Nations Unies avec les versements effectifs représentant respectivement $43 \%, 22,9 \%, 12,0 \%, 10,7 \%$ et $8,6 \%$ du total des versements effectifs. Du côté bilatéral, on peut retenir le Royaume-Uni, les Etats unies, la Belgique, la chine et l'Allemagne comme grands donateurs bilatéraux avec respectivement $30,5 \%, 18,5 \%, 13,3 \%, 8,1 \%$ et $6,8 \%$ de participation effective sur un montant de 6,7 Milliards de dollars US (Ministère des Affaires Etrangères, 2020). En dépit de ce qui précède, force est de constater que beaucoup d'indicateurs socioéconomiques que cible l'APD ne connaissent pas une évolution favorable et significative en RDC. Le PIB par habitant est I'un de plus faible au monde (moins de 500 dollars américains) (PNUD, 2016 ; BCC, 2018 ; Banque Mondiale, 2018). Entre 1990 et 2018 le revenu national brut (RNB) par habitant a diminué d'environ 61.1 \% (PNUD, 2019). Les inégalités sociales se creusent davantage et amplifiées actuellement par la pandémie de COVID-19 encours (Murhula et al., 2020 ; Balasha et al, 2020) et plus de 26 millions de Congolais vivaient encore dans l'extrême pauvreté en 2015 soit $7 \%$ des pauvres de la planète, ce qui place ce pays dans le top 5 des pays les plus pauvres (Kasongo et Ditend , 2020). Entre 2015 et 2019, le nombre de personnes souffrant de l'insécurité alimentaire aiguë est passé de 5,9 à 15,6 millions de personnes, soit une hausse de 164,40\% (Ministère de Plan 2020). L'accès aux soins décents et à l'eau ainsi qu'à l'électricité est resté une bête noire pour la population congolaise (Lubangu, 2018). Le taux d'accès de la population à l'électricité est estimé à $6 \%$ malgré son gigantesque potentiel hydroélectrique évalué à $13 \%$ du potentiel mondial et dont la production est en très grande partie destinée à l'exportation. Seulement $22 \%$ de la population accède à l'eau potable, alors que son réseau hydrographique représente le 1/4 des ressources en eau douce du continent africain (RDC, 2006 cité par Tshimanga, 2012). L'objectif de cette étude était d'évaluer l'impact de l'aide publique sur la croissance économique et la réduction de la pauvreté dans un pays pauvre. Nous nous sommes intéressés au cas spécifique de la République démocratique du Congo, pays bénéficiaire de l'APD pour répondre à deux questions principales : (i) quel est le niveau de dépendance de la RD Congo à l'aide publique au développement ? (ii) quel est l'impact de l'aide publique au développement sur la croissance économique et la réduction de la pauvreté ? Nous émettons l'hypothèse que la RDC connait une forte dépendance à l'égard de l'APD et celle-ci aurait un effet positif sur la croissance économique et la réduction de la pauvreté. Ce travail poursuit trois objectifs spécifiques : (1) déterminer le degré de dépendance à l'égard de l'APD ; (2) mesurer et analyser l'impact de l'aide publique au développement sur la croissance économique et la réduction de la pauvreté en RDC; (3) fournir des pistes des solutions éventuelles pour améliorer l'efficacité de l'APD en RD Congo.

\section{Revue de la littérature}

\subsection{La théorie de Big push}

Les travaux de Rosenstein Rodan (1943) "les problèmes de l'industrialisation de l'Europe de l'Est et du Sud-Est » ont donné naissance à la théorie du Big push selon laquelle, la solution pour sortir les pays pauvres de leur trappe à pauvreté serait d'y investir massivement via l'aide internationale. Rosenstein souligne en substance que des apports massifs en capitaux extérieurs doivent permettre aux pays pauvres de financer leurs investissements et de brûler des étapes préalables au décollage. Sachs Jeffrey s'aligne à cette approche et défend l'idée d'une trappe de la pauvreté, de laquelle un pays pauvre ne peut se sortir sans un apport financier extérieur (Sachs, 2005). En opposition, la vision plus extrémiste, (Easterly, 2009 ; Moyo, 2009a) se sont attaqués à l'idée du Big Push avec des arguments tirés de la tradition du choix public. Easterly qui s'oppose à Sachs, avait montré en 2009 que l'aide n'achète pas la croissance, et cela indépendamment du montant des sommes déboursées. II a insisté sur l'existence de deux scandales dans le monde à savoir : (1) l'existence de plusieurs pauvres dans un monde riche et (2) la somme déboursée d'aide au développement (soit 2300 milliards) depuis un demi-siècle n'a produit que peu de résultat (Easterly, 2009). Selon lui, l'aide se borne à élargir et à renforcer les pouvoirs centraux. Pourtant Stiglitz (2002) pense que, même si l'aide n'a pas toujours fonctionné, elle a joué un rôle stratégique dans la croissance et la lutte contre la misère dans nombreux pays en voie de développement. Dambisa Moyo n'est pas aussi d'accord des arguments 
avancés par Sachs et Stiglitz. Dans son ouvrage "Aide fatale ", le souhait de Moyo (2009) n'est que la mort de l'aide du fait qu'elle encourage la corruption, ralentit la croissance et décourage la libre entreprise. Moyo et Easterly rendent l'aide responsable quasiment de tous les maux de l'Afrique, en allant jusqu'à dire qu'elle a contribué à rendre les pauvres encore plus pauvres (Moyo, 2009a ; Easterly, 2009). Moyo a beaucoup insisté dans son ouvrage sur la nécessité d'ouvrir l'Afrique au commerce mondial, de supprimer les subventions aux agriculteurs européens et américains qui concurrencent déloyalement les agriculteurs des pays aidés. Ces critiques à l'encontre de l'aide ne datent pas d'aujourd'hui. Peter Bauer (1972) estimait que l'aide se limite à enrichir les élites des pays bénéficiaires et dans une remarque ironique restée célèbre, déclara : " l'aide est un processus par lequel les pauvres des pays riches subventionnent les riches de pays pauvres ».

\subsection{La théorie du cercle vicieux}

Dans les années 50, Ragnar Nurske a avancé l'idée selon laquelle les pays sous-développés se trouveraient dans cette spirale qu'il a appelé "Cercle vicieux de la pauvreté " qui donne beaucoup de difficulté pour amorcer le développement économique (Nurske , 1953). Cette approche voit dans la pauvreté la cause essentielle du sous-développement (Kitsali K., 2013). Au vu de la figure 1, Nurske a formulé une célèbre citation selon laquelle "un pays est pauvre parce qu'il est pauvre». Celle-ci ne reflèterait qu'une idée commune : le développement est une question d'argent (Perkins D. et al. 2014). Dans ce contexte, le financement extérieur est un besoin absolu pour casser ce cercle vicieux et pallier à l'insuffisance de l'épargne et déclencher le processus d'investissement (Kitsali K., 2013). La figure 1 montre comment s'entretient la pauvreté dans les pays en voie de développement (PVD).

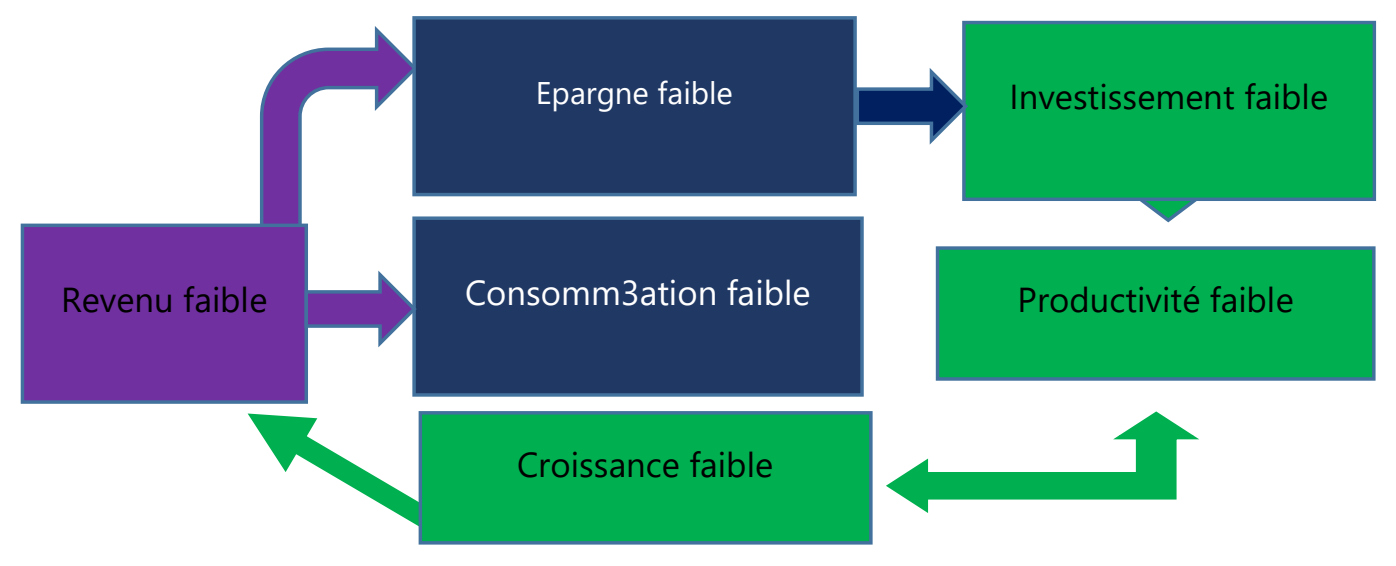

Figure 1. Cercle vicieux de la pauvreté, élaboré par les auteurs sur base de la théorie économique

\section{3 héorie néoclassique de Rostow}

Comme les deux précédentes théories, Walt Rostow justifie le sous-développement des PVD par l'insuffisance de l'épargne intérieure. En fait, il voit dans l'aide un outil de financement des investissements et d'augmentation des réserves de capital dans les pays en développement (Rostow W.1960). Comme l'épargne intérieure est insuffisante pour réaliser un niveau donné de croissance, le pays devrait faire appel aux capitaux étrangers, c'est-à-dire aux aides. Le pays devrait utiliser ces aides pour investir et obtenir de la croissance, mais il doit les utiliser efficacement.

\section{4 Le modèle de double déficit de Chenery et Strout}

Le modèle de double déficit (two-gap) a été développé au milieu des années 1960. L'idée de base était que le processus de croissance des pays les moins avancés était freiné par une épargne domestique et des réserves en devises trop faibles (double déficit) (Chenery et Strout, 1966). D’après eux, les exportations constituent une source de devises permettant de financer les importations. Ainsi, un déficit du commerce extérieur se traduit par une insuffisance des ressources nécessaires pour le financement des importations. Il est alors très vite apparu comme évident qu'une aide financière (comblant ces déficits) de la part des pays industrialisés ayant, eux, réalisé leur décollage économique, permettrait de favoriser celui des pays en voie de développement. 


\subsection{Quelques résultats empiriques}

La problématique de l'efficacité de l'aide revient à l'ordre du jour de la plupart des rendez-vous internationaux, justement car il s'agit d'un élément central des enjeux économiques du monde actuel. Le problème de l'efficacité de l'aide publique à promouvoir la croissance et à réduire la pauvreté fait l'objet d'un grand nombre de travaux des chercheurs aboutissant à différentes conclusions que nous tentons de regrouper en trois grandes catégories: (i) la relation entre aide-croissance est négative; (ii) la relation entre aide et croissance positive et maximise de la réduction de la pauvreté (iii) enfin, l'efficacité de l'aide sur la croissance est conditionnelle.

\subsection{L'aide n'influe pas sur la croissance et peut même la freiner}

Griffin et Enos (1970) ont été parmi les premiers à remettre en question l'efficacité de l'aide dans une étude empirique, qui a fait état d'une corrélation simple négative entre l'aide et la croissance dans 27 pays. Boone (1994) a conclu qu'il y a absence de corrélation positive entre l'aide et la croissance, bien que les résultats de son étude aient été sujets à des critiques (qualité douteuse). Ces mêmes conclusions ont été rapportées par Mosley P. (1980); Radelet et al. (2004) montrant que la relation entre l'aide et la croissance était quasi inexistante. Selon ces chercheurs, l'aide ne favorise pas la croissance pour plusieurs raisons : (i) l'aide est engloutie dans les limousines et les palais présidentiels; (ii) elle pourrait encourager la corruption; (iii) l'aide enlève la raison d'accroissement de la productivité du secteur privé ; (iv) l'aide peut entraîner une appréciation de la monnaie et ainsi diminuer la rentabilité de la production des biens échangeables (le syndrome hollandais); (vi) l'aide alimentaire peut être à l'origine d'une baisse du prix des produits agricoles et du revenu des producteurs ; (vii) les flux d'aide peuvent réduire l'épargne des particuliers (en influant sur les taux d'intérêt) et l'épargne publique (en se répercutant sur les recettes de l'État); (viii) l'aide peut aider les mauvais gouvernements à se maintenir au pouvoir, ce qui perpétue les mauvaises politiques économiques et retarde les réformes.

\subsubsection{La relation positive entre aide, croissance et pauvreté avec rendement décroissant}

Plusieurs auteurs ont constaté une relation positive entre l'aide et la croissance (par exemple Papenek, 1973; Levy, 1988), ce qui a donné lieu à des échanges animés avec les chercheurs qui avaient conclu à l'absence d'une telle relation (Radelet $\mathrm{S}$. et al., 2004). Hansen H. et Tarp F. (2001) ont mené des études durant les trente années qui précédaient l'an 2000 et avaient abouti aux conclusions suivantes : l'aide accroît l'épargne nationale, l'investissement, elle a un effet positif sur la croissance. Gomanee et al. (2005) suggèrent une influence positive de l'aide sur l'indicateur de développement humain et sur la réduction de la mortalité infantile, effet qui passe par le financement de dépenses publiques favorables aux plus pauvres, par exemple la construction et/ou l'équipement des hôpitaux, routes de dessertes agricoles. Cependant, même si l'effet est positif, elle n'est pas linéaire et cela est dû à la faible capacité d'absorption des pays qui ont reçu les aides (Collier $P$. et Dollar D. 2002 ; Clemens et al., 2004). Par ailleurs, la plupart d'études récentes qui admettent le rendement décroissant ont conclu en faveur d'une relation positive (Dalgaard et al., 2004 ; Hansen et Tarp, 2004) et que l'aide a un rendement décroissant sur la croissance économique. En effet, dépasser un certain seuil, un dollar d'aide supplémentaire aurait moins d'impact sur la croissance ou la pauvreté que le dollar précédent. Ce seuil se situerait à peu près à $25 \%$ du PNB du pays qui reçoit l'aide (Hansen et Tarp , 2004). Clemens et al. (2004) ont conclu que l'aide a un effet absolument nul sur la croissance dès qu'elle atteint $8 \%$ du PNB du pays bénéficiaire, et un effet négatif sur la croissance au-delà de ce taux.

\subsubsection{La relation entre l'aide et la croissance est conditionnelle}

Après le développement des modèles " two-gap » de Chenery et Strout dans les années 1960, le débat s'était essoufflé. II a été relancé à la fin des années 1990, lorsque Burnside et Dollar ont fait des publications des résultats pouvant apparaître embarrassants. Burnside et Dollar (1997) ont conclu que l'aide stimulait la croissance uniquement dans les pays qui adoptaient des politiques économiques judicieuses. D'autres chercheurs, ont identifié d'autres caractéristiques susceptibles d'influer sur la relation aide-croissance. Collier et Dehn, (2001) ont indiqué les chocs de prix des exportations alors que (Guillaumont et Chauvet, 2001 ; Amprou, et Chauvet, 2004) ont dévoilé les perturbations climatiques et les termes de l'échange. La conclusion des investigations menées par Collier et Dollar a également montré que la qualité des politiques économiques et la qualité des institutions étaient au centre de l'efficacité de l'aide (Collier P. et Dollar D. 2002). L'attrait de cette approche tient au fait qu'elle peut expliquer pourquoi l'aide semble d'une part, avoir favorisé la croissance dans des pays tels que la Corée, le Botswana, l'Indonésie et, récemment, le Mozambique et l'Ouganda et d'autre part, ne pas avoir stimulé la croissance dans des pays comme l'Haïti, le Libéria, le Congo, la RDC et les Philippines (Radelet et al., 2004). Cette étude s'aligne à la deuxième approche (la relation entre aide-croissance-pauvreté est généralement positive bien que le rendement décroisse à mesure que l'aide augmente) et se démarque de toutes ces études du fait qu'elle emprunte un champ nouveau (la RD Congo). 


\section{Milieu d'étude et méthodes}

\subsection{Brève description de la République Démocratique du Congo}

Située au centre de l'Afrique, la République démocratique du Congo est le deuxième pays le plus vaste du continent africain après l'Algérie avec une superficie de plus de $2.345 .409 \mathrm{~km}^{2}$ (Ministère de Plan, 2020). Elle partage 9165 kilomètres de frontière avec neuf pays voisins, à savoir : la République du Congo, l'Ouganda, le Burundi, le Rwanda, la Tanzanie, la République Centrafricaine, le Soudan ainsi que la Zambie et l'Angola (Figure 2).

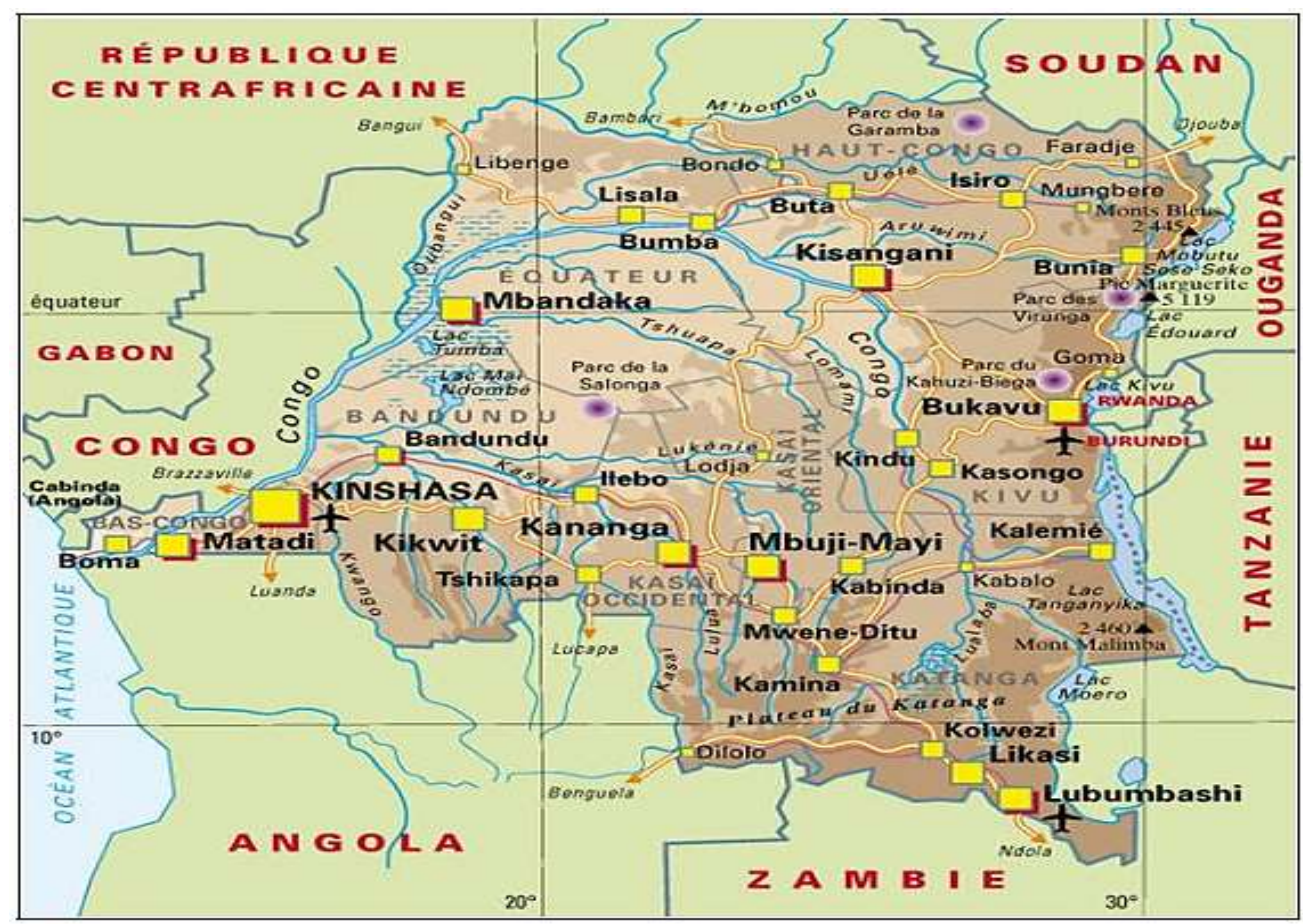

Figure 2. Carte administrative de la RDC tirée du Ministère de santé publique, 2011

En 2019, la population de la RDC a été estimée autour de 86,79 millions d'habitants (Ministère de Plan, 2020) contre 77 millions d'habitants en 2016 dont la majorité $(61,2 \%)$ vivait dans les milieux ruraux (PNUD, 2016). Cet accroissement est jugé préoccupant et même préjudiciable au développement économique du pays face à la forte demande sociale qui peut en découler en termes d'éducation, de soins de santé, de logement, de transports, etc. Sur le plan social, les indicateurs ont des niveaux préoccupants. A titre illustratif, la RDC est très mal classée sur l'Indice du Développement Humain (Ministère de Plan, 2020 ; PNUD, 2019). L'IDH s'établit à 0.459 en 2018, ce qui place le pays dans la catégorie développement humain faible et au $179^{\mathrm{eme}}$ rang parmi 189 pays et territoires (PNUD, 2019). Le contexte actuel marqué par la crise sanitaire de la covid-19, avec des effets néfastes sur la croissance et le bien-être collectif. Le défi immédiat pour la RDC est de contenir la crise sanitaire, de réduire l'incertitude politique et d'amoindrir les déséquilibres du cadre macroéconomique déjà préoccupant. L'économie congolaise a subi une réelle hémorragie suite à la propagation rapide et généralisée de la pandémie covid19 qui a conduit à : (i) un repli de la croissance économique ; (ii) un creusement du déficit public ; (iii) une dépréciation considérable du taux de change et une accélération de l'inflation avec comme corollaire la baisse du pouvoir d'achat ; (iv) une contraction des échanges commerciaux et des réserves de change ; (v) une augmentation du chômage et du sous-emploi.

\subsection{Source de données et justification de la période d'étude}

Les données que nous utilisons dans cette étude proviennent de la base des données de la banque mondiale disponible sur api.worldbank.org/v2/fr/country/COD?downloadformat=excel et des rapports annuels de la banque centrale du Congo (BCC). En effet, la qualité des données issues de ces institutions est sujette à controverses. Les informations fournies par ces institutions sont souvent discordantes et peuvent s'avérer insuffisantes dans le sens où elles ne révèlent pas l'effort global du monde extérieur en faveur de la RDC ni la traçabilité quant à l'usage effectif de tous les fonds reçus. La période de l'étude s'étale de 1990 à 2018 et se justifie par le souci de couvrir un nombre suffisant d'années pour dégager des tendances plus ou moins significatives. En outre, l'année 1990 est prise comme point de départ car c'est durant cette période que les problèmes 
liés l'APD se sont manifestés avec grand éclat accouchant les travaux de Burnside qui préconisaient que l'allocation de l'aide soit conditionnelle à la bonne gouvernance afin d'améliorer son efficacité. L'année 2018 est motivée par la disponibilité des données publiées par la Banque Mondiale et la Banque Centrale du Congo.

\subsection{Spécification des modèles}

\subsubsection{Modèle de croissance économique}

La présente étude s'appuie sur le modèle développé par Burnisde et Dollar (1997) pour évaluer l'impact de l'aide publique sur la croissance économique. Le modèle économétrique utilisé dans ce travail à des fins d'estimation adapté aux spécificités de la RD Congo peut prendre la forme linéaire suivante :

$$
G_{t}=\beta_{0}+\beta_{1} S_{t}+\beta_{2} A P D_{t}+\beta_{3} I D E_{t}+\beta_{4} D E F B_{t}+u_{t}
$$

Où $\mathrm{G}$ est le taux de croissance économique; $\mathrm{S}$ est l'épargne intérieure en pourcentage du PIB ; APD est l'aide publique au développement reçue par habitant; DEFB est le déficit budgétaire en pourcentage du PIB (variable de la politique économique) et IDE est l'investissement direct étranger en pourcentage du PIB. Les $\beta$ représentent les coefficients des variables explicatives; t est l'indice de temps et u le terme d'erreur.

\subsubsection{Modèle de réduction de pauvreté}

Le modèle de réduction de la pauvreté est inspiré de Gomanee et al (2003) où le niveau de la pauvreté est fonction de : (i) PIB par habitant ( $\mathrm{Y}$ ) ; (ii) les dépenses gouvernementales (G); et (ii) l'aide extérieure (A). Dans ce travail, pour capter le niveau de pauvreté, nous avons retenu l'espérance de vie à la naissance comme variable expliquée. Le modèle peut s'écrire de la manière suivant :

$$
E S P_{t}=\beta_{0}+\beta_{1} P I B_{t}+\beta_{2} A P D_{t}+\beta_{3} D E P U B_{t}+\beta_{4} T B S+u_{t}
$$

Où ESP est l'espérance de vie à la naissance ; PIB est le produit intérieur brut réel par habitant ; DEPUB représente les dépenses gouvernementales et TBS est le taux brut de scolarité. En effet, ce modèle permet de mesurer les implications directes de l'APD sur l'espérance de vie à la naissance, subséquent à la réduction de la pauvreté. Il est également possible au travers ce modèle de voir si la croissance est effectivement un puissant canal de réduction de la pauvreté.

\subsection{Mesure du degré de dépendance à l'égard de l'APD}

L'évaluation de la dépendance à l'égard de l'APD s'est basée sur la méthode de Mandela R. et Naoutem (2009). Les deux formules retenues peuvent s'écrire de la manière suivante :

$$
\text { Ration }=\frac{A P D}{P I B} * 100 ; \text { Ration }=\frac{A P D}{\text { Population totale }}
$$

Où APD est l'aide publique au développement et PIB est le produit intérieur brut. Les données utilisées pour ces calculs sont : (i) Le PIB courant (en dollar américain) ; (ii) APD courant nette reçue (en dollar américain) et (iii) la population (en million d'habitants). Toutes ces données proviennent essentiellement de la base des données de la Banque Mondiale.

\section{Résultats et Discussion}

\subsection{Analyse de la dépendance à l'égard de l'APD}

Le résultat présenté au tableau 1 montre que, l'APD reste une ressource externe indispensable dans l'économie congolaise.

\begin{tabular}{|c|c|c|c|c|c|}
\hline \multicolumn{3}{|c|}{ Ratio APD/PIB } & \multicolumn{3}{|c|}{ Ratio APD/Population } \\
\hline \multirow{2}{*}{$\begin{array}{c}\text { Moyenn } \\
\text { e }\end{array}$} & \multicolumn{2}{|c|}{ 95\% Intervalle de confiance } & \multirow{2}{*}{$\begin{array}{c}\text { Moyenn } \\
\text { e }\end{array}$} & \multicolumn{2}{|c|}{$95 \%$ Intervalle de confiance } \\
\hline & Minimum & $\begin{array}{c}\text { Maximu } \\
\mathrm{m}\end{array}$ & & Minimum & $\begin{array}{c}\text { Maximu } \\
\mathrm{m}\end{array}$ \\
\hline $9,7 \%$ & $0,9 \%$ & $60,6 \%$ & 26,8 & 2,8 & 105,3 \\
\hline
\end{tabular}
Dans l'intervalle de confiance de $95 \%$, I'APD a représenté en moyenne près de $10 \%$ du PIB et un congolais a reçu en moyenne, près de 27 dollars américains d'aide publique au développement entre 1990 et 2018 (Tableau 1).

Tableau 1. Degré de dépendance de la RD Congo à l'égard de l'APD 
C'est depuis l'an 2000 que l'aide publique a connu une reprise fulgurante en RD Congo (Figure 3). Elle a atteint en 2003 un record historique de plus de $60 \%$ du PIB, soit plus de 105 dollars par habitant. Cette hausse résulte de la reprise de la coopération structurelle avec les institutions internationales (Banque mondiale, FMI, etc.).

\section{Ratio APD sur PIB}

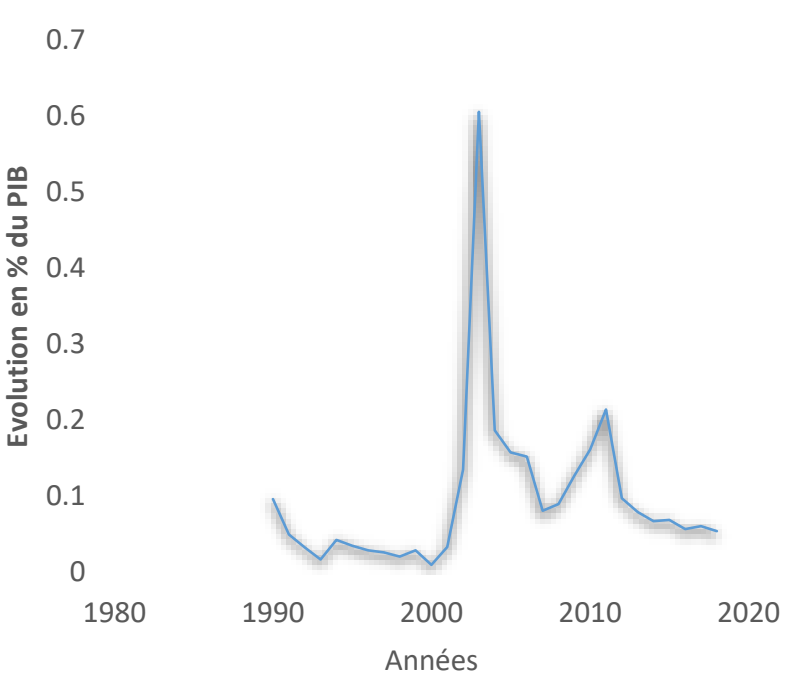

Ratio APD par habitant

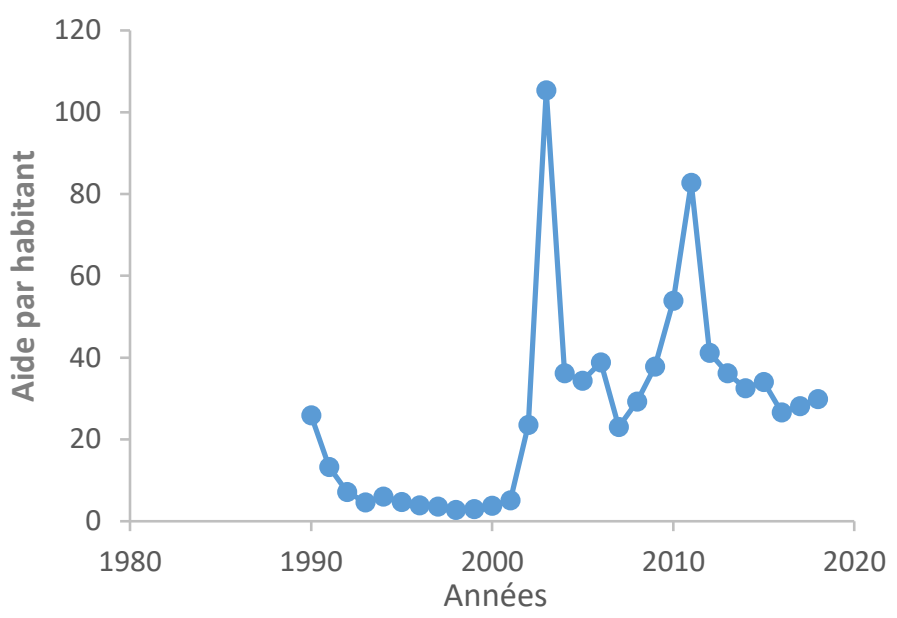

Figure 3. Evolution du niveau dépendance à l'aide publique au développement en RD Congo

Le résultat relatif à l'aide publique par habitant montre la sur-dépendance de la RDC à l'aide et donc une forte contrainte des conditions imposées par les bailleurs de fonds. Ce résultat est un signal d'une forte dépendance à l'égard de l'aide extérieure subséquent à l'incapacité pour la RDC à financer ses propres stratégies de développement. Plusieurs auteurs dont Griffin et Enos (1970); Moyo (2009b) ont montré que la dépendance vis-à-vis de l'aide est désavantageuse pour l'économie car elle tend à saper la qualité de la gouvernance, en encourageant la corruption et en provoquant des conflits sur le contrôle des fonds d'aide. L'étude de Gueye (2006) a montré que les pays africains comptaient parmi les bénéficiaires d'aide les plus importants au monde et beaucoup d'entre eux recevaient un montant net d'aide au développement qui équivaut à $10 \%$ de leur Produit National Brut (PNB).

\subsection{Relation aide publique et croissance économique}

Le tableau ci-dessous présente les résultats de l'estimation de la relation directe entre aide publique et la croissance économique. Le coefficient associé à la variable APD est positif et significativement différent de zéro (0.070648) (Tableau 2), ce qui signifie que l'aide est porteuse de la croissance.

Tableau 2. Résultats détaillés de la régression de la relation directe aide-croissance

\begin{tabular}{|c|c|c|c|c|}
\hline \multicolumn{5}{|c|}{ Dependent Variable: G } \\
\hline \multicolumn{5}{|c|}{ Method: Least Squares } \\
\hline \multicolumn{5}{|c|}{ Date: 20/11/20 Time: 06:56 } \\
\hline \multicolumn{5}{|c|}{ Sample: 1990- 2018} \\
\hline \multicolumn{5}{|c|}{ Included observations: 29} \\
\hline Variable & Coefficient & Std. Error & t-Statistic & Prob. \\
\hline $\mathrm{C}$ & 0.219337 & 1.989397 & 0.110253 & 0.9131 \\
\hline APD & 0,070648 & 0.034020 & 2.076656 & 0.0487 \\
\hline DEFB & 2.189305 & 0.712065 & 3.074588 & 0.0052 \\
\hline IDE & 0.238272 & 0.254156 & 1.937503 & 0.0358 \\
\hline$S$ & $4.85 \mathrm{E}-10$ & $3.08 \mathrm{E}-10$ & 1.573888 & 0.1286 \\
\hline R-squared & 0.730998 & \multicolumn{2}{|c|}{ Mean dependent var } & 0.049721 \\
\hline
\end{tabular}




\begin{tabular}{|l|l|l|l|}
\hline Adjusted R-squared & 0.686164 & S.D. dependent var & 0.052813 \\
\hline S.E. of regression & 0.018912 & Akaike info criterion & -5.532372 \\
\hline Sum squared resid & 0.009004 & Schwarz criterion & -5.768113 \\
\hline Log likelihood & 75.21940 & Hannan-Quinn criter. & -5.606203 \\
\hline F-statistic & 3.637055 & Durbin-Watson stat & 2.127919 \\
\hline Prob(F-statistic) & 0.000401 & & \\
\hline
\end{tabular}

Ces résultats indiquent que, une augmentation dix millions de dollars de l'aide publique au développement entraîne, ceteris paribus, une augmentation du taux de croissance économique (G) d'environ 0,7 \% en RDC. Cet effet est expliqué par le fait que l'aide est allouée au niveau de l'investissement productif qui a un impact direct sur la croissance économique. Les résultats de l'étude de Tang et Bundhoo (2017) sur dix pays de l'Afrique Subsaharienne ont montré que l'aide publique était un bon ingrédient pour compléter les besoins d'investissement et d'importation dans la région. En réalité, une relation positive entre aide publique et croissance économique est toujours encourageante car plus d'aide implique une croissance économique plus élevée. Les résultats que nous avons trouvés dans cette étude sont en nette conformité avec l'étude de Gueye (2006) qui a trouvé un coefficient de $(0,73)$ entre l'aide et le taux de croissance au Sénégal pour une augmentation d'un million de dollars d'aide. En revanche, ils contredisent ceux obtenus par Griffin et Enos (1970); Mosley (1980); Boone $(1994,1996)$ qui ont trouvé une relation négative entre aide extérieure et croissance dans plusieurs pays en voie de développement.

\subsection{Relation entre l'aide et la réduction de la pauvreté}

Le tableau 3 présente les résultats de l'estimation de la relation entre aide publique et espérance de vie à la naissance (ESP). Les résultats montrent que l'aide publique exerce une influence positive et significative sur l'espérance de vie à la naissance. Une augmentation de dix millions dollars de l'aide publique entraine une augmentation de l'espérance de vie d'environ 0,4 ans (soit quatre mois et neuf jours) en RD Congo (Tableau 3).

Tableau 3. Résultats détaillés de la régression de la relation directe aide-pauvreté

\begin{tabular}{|c|c|c|c|c|}
\hline \multicolumn{5}{|c|}{ Dependent Variable: ESP } \\
\hline \multicolumn{5}{|c|}{ Method: Least Squares } \\
\hline \multicolumn{5}{|c|}{ Date: $20 / 11 / 20$ Time: $19: 05$} \\
\hline \multicolumn{5}{|c|}{ Sample: 1990- 2018} \\
\hline \multicolumn{5}{|c|}{ Included observations: 29} \\
\hline Variables & Coefficient & Std. Error & t-Statistic & Prob. \\
\hline $\mathrm{C}$ & -2.05058 & 3.093579 & -1.09301 & 0.2100 \\
\hline $\mathrm{PIB}$ & -0.007687 & 0.018214 & 3.824317 & 0.0206 \\
\hline APD & 0.039462 & 0.017824 & 2.213983 & 0.0366 \\
\hline DEPUB & $-1.75 \mathrm{E}-09$ & $2.44 \mathrm{E}-09$ & -0.717137 & 0.4802 \\
\hline TBS & $\begin{array}{l}0 \\
.162410\end{array}$ & 0.023039 & 7.049435 & 0.0000 \\
\hline R-squared & 0.829747 & \multicolumn{2}{|c|}{ Mean dependent var } & 0.056152 \\
\hline Adjusted R-squared & 0.801372 & \multicolumn{2}{|c|}{ S.D. dependent var } & 0.169395 \\
\hline S.E. of regression & 0.008203 & \multicolumn{2}{|c|}{ Akaike info criterion } & -4.232683 \\
\hline Sum squared resid & 0.07007 & \multicolumn{2}{|c|}{ Schwarz criterion } & -4.468423 \\
\hline Log likelihood & 82.37390 & \multicolumn{2}{|c|}{ Hannan-Quinn criter. } & -4.306514 \\
\hline F-statistic & 3.24176 & \multirow{2}{*}{\multicolumn{2}{|c|}{ Durbin-Watson stat }} & 2.350800 \\
\hline Prob(F-statistic) & 0.001058 & & & \\
\hline
\end{tabular}

En effet, selon le modèle classique, la réduction de la pauvreté dans les PVD passe avant tout par une croissance économique beaucoup plus rapide. Ainsi, si l'aide exerce une influence positive sur la croissance économique, elle contribue par ce canal à la réduction de la pauvreté. Cependant, ce raisonnement repose sur l'hypothèse que l'aide n'a pas d'effet direct sur la pauvreté et que son effet passe essentiellement par la croissance (Amprou et Chauvet, 2004). Cette approche est remise en cause par les résultats de cette étude, qui soulignent un effet directement positif de l'APD sur l'espérance de vie à la 
naissance sans emprunter le canal de la croissance économique. La croissance économique exerce une influence négative sur l'espérance de vie en RDC. Le coefficient associé au PIB réel par habitant est négatif et significativement diffèrent de zéro (0.007687). II implique qu'une augmentation de $10 \%$ produit intérieur brut réel par habitant entraine, toute chose restant égale par ailleurs, une diminution de l'espérance de vie à la naissance d'environ 0,07 ans (soit vingt-cinq jours). Pour notre part, nous pouvons expliquer cette situation de la manière suivante : depuis l'an 2000, la croissance élevée en RDC est réelle, mais non inclusive. Elle est portée essentiellement par les secteurs d'exportation, profite aux capitaux extérieurs, ne crée pas d'emplois sécurisants, décents et significatifs, ce qui fait qu'elle a un effet réduit sur la pauvreté. Matata (2018), avait montré aussi que la croissance en RD Congo était remarquable sur le plan quantitatif mais illusoire et déficitaire sur le plan qualitatif. PNUD (2017) avait indiqué également que la croissance en RDC n'avait pas d'effets notables dans la lutte contre la pauvreté de masse. Ces arguments convergent avec ceux de plusieurs auteurs dont Perkins D. et al. (2014) ; Stiglitz (2012) qui ont montré que le niveau de la croissance économique n'est encore suffisant pour permettre une réduction drastique de la pauvreté à cause de la montée des inégalités dans les pays en développement.

\section{Conclusion}

La République démocratique du Congo a depuis longtemps bénéficié de l'aide publique au développement de la part de ses partenaires multilatéraux et bilatéraux qui l'accompagnent dans ses objectifs de croissance économique et de réduction de la pauvreté. Ce travail a mesuré et analysé les effets de l'aide publique au développement sur la croissance et la réduction de la pauvreté en RD Congo. Les résultats ont mis en évidence la forte dépendance du pays à l'aide publique au développement et celle-ci a théoriquement contribué positivement à la croissance économique et à la réduction de la pauvreté en République Démocratique du Congo. Cependant, les observations directes sur terrain et nombreux travaux soutiennent que la croissance économique cache encore beaucoup d'inégalités et de la misère dans la société congolaise. L'amélioration de la gestion de l'aide et l'harmonisation des procédures des bailleurs constituent les solutions essentielles pour rendre l'aide publique plus efficace. Six recommandations sont assorties de cette étude pour que l'APD donne des résultats fructueux en RDC: (i) harmoniser ses plans d'action avec ceux des bailleurs pour permettre une plus grande efficacité de l'aide publique au développement; (ii) allouer l'aide publique au développement reçue aux infrastructures de base susceptibles à stimuler la croissance économique ; (iii) orienter l'APD vers les secteurs pro-pauvres. Les secteurs pro-pauvres (par exemple l'agriculture, l'artisanat, la culture, etc.) sont ceux dont la croissance profite aux pauvres et dont les acteurs sont essentiellement constitués des populations vulnérables (Koudougou L. 2012). Il s'agira principalement de promouvoir des innovations à fortes externalités positives dans ces secteurs. Le gouvernement pourra par exemple rendre accessibles aux paysans, les innovations techniques issues des institutions de recherches, les faire participer à la transformation du mode de production paysan et à la compétitivité des produits agricoles (PNUD, 2016) ; (iv) intensifier les relations de coopération avec les partenaires au développement intervenant dans le secteur de l'éducation. Les résultats de la régression du modèle de pauvreté montrent une augmentation hautement significative de l'espérance de vie à mesure que le taux brut de scolarité s'accroit, ce qui confirme que le secteur de l'éducation est porteur de la contraction de la pauvreté. (v) créer un comité de suivi de l'aide publique au développement à l'assemblée nationale afin de la contrôler et d'accroitre davantage son efficacité ; (vi) prendre des décisions ambitieuses en direction de l'amélioration du cadre institutionnel afin de rendre plus productive l'aide publique au développement. Les recherches ultérieures pour compléter cette étude pourront évaluer la capacité d'absorption et la fongibilité mais aussi étudier la relation existante entre APD et la démocratie, APD et l'intégration régionale.

\section{Références}

[1] Amprou, J. et Chauvet L. (2004). Efficacité et Allocation de l'aide: Revue des Débats. Notes et Documents 6, Agence Française de Développement, Paris.

[2] Banque, M. (2015). Renforcer la résilience de long terme de la RDC: le Rôle de la dédollarisation, de la prospection artisanale et de la diversification économique. Rapport de suivi de la situation économique et financière, Troisième Edition.

[3] Banque, M. (2016). Choc Exogène, Stabilité Macroéconomique et Développement : Options de Politique Économique. Rapport de Suivi de la Situation Économique et Financière en République Démocratique du Congo, 4 eme Édition.

[4] Bauer P. (1987). Creating the Third World: Foreign Aid and its Offspring. Journal of Economic Growth, 2(4).

[5] Bonne, P. (1996). Politics and the Effectiveness of Foreign Aid. European Economic Review, 40(2),289-329.

[6] Boone, P. (1994). The impact of foreign aid on savings and growth, Centre for Economic Performance Working Paper No. 677, London School of Economics.

[7] Burnside C. et Dollar, D. (2000). Aid, policies, and growth, American journal review 90, 847-868.

[8] Burnside, C. et Dollar, D. (1997). Aid, policies, and growth. The World Bank Policy Research

[9] Chauvet, L. et Guillaumont, P. (2001). Aid and Performance: a Reassessment. Journal of Development Studies, 66-92.

[10] Chenery, B. et Strout, M. (1966). Foreign Assistance and Economic Development. The American Economic Review, 679-733. 
[11] Collier, P. et Dehn, J. (2001). Aid, Shocks, and Growth. The World Bank Working Paper.

[12] Clemens, M., Radelet, S., et al. (2004). Counting Chickens When They Hatch : Timing and the Effects of Aid on Growth. Working Paper, 44, Centre for Global Development.

[13] Dalgaard, C-J., Hansen, H. et Tarp, F. (2004). On the empirics of foreign aid and growth, Economic Journal, $191-216$.

[14] Easterly, W. (2009). Le fardeau de l'homme blanc : l'échec des politiques occidentales d'aide aux pays pauvres, traduit de l'anglais par Patrick Hersant.

[15] Gomanee, K., Morrissey, O., Mosley, P. et Verschoor, A. (2005). Aid, government expenditure, and aggregate welfare. World Development 33, 355-370.

[16] Griffin, K. et Enos, J. (1970). Foreign Assistance: Objectives and Consequences. Economic Development and Cultural Change, 18, 313327.

[17] Gueye, F. (2006). Efficacité de I'Aide Publique au Développement: cas du Sénégal, DEA, Université Cheikh Anta Diop de Dakar.

[18] Hansen, H. et Tarp, F. (2001). Aid and growth regression, journal of development Economics, 64, 547-570.

[19] Kasongo, N. et Ditend Y. (2020). Les pratiques du mix marketing et le développement durable en République Démocratique du Congo: Du marketing classique au marketing sociétal, International Journal of Multidisciplinary and Current Research, 501-508.

[20] Kitsali, K., 2013. Modèle de fonctionnement des exploitations familiales pour le développement agricole et rural du Katanga (Cas de la zone agricole de Sambwa) [Thèse de doctorat] Université de Lubumbashi.

[21] Levy, V. (1988). Aid and Growth in sub-Saharan Africa: The Recent Experience, European Economic Review, 1777-1795.

[22] Lubangu, E. (2018). Conjoncture économique et pratiques de don en période électorale. Une contextualisation congolaise de la pensée de Marcel Mauss, Edilivre.

[23] Mandela, R. et Naoutem, B. (2009). L’Aide Publique au Développement en République Démocratique du Congo : La spécificité de l'aide aux Etats fragiles ». Master 2 gestion de projets de développement en Afrique.

[24] Matata, P. (2018). Plaidoyer pour la bonne gouvernance et le développement. Recueil des discours de la primature 2012-2016, Editions Michel Lafon.

[25] Ministère de plan (2020). Rapport d'examen national volontaire des objectifs de développement durable, https://sustainabledevelopment.un.org/content/documents/26297VNR_2020_DRC_Report_French.pdf.

[26] Ministère de santé publique (2011). Rapport narratif : profil pharmaceutique de la République Démocratique du Congo 2011.

[27] Ministère des Affaires Etrangère (2020). Rapport National d'évaluation de la mise en œuvre du Programme d'Action d'Istanbul en faveur des PMA pour la décennie 2011-2020 en République Démocratique du Congo.

[28] Mosley, P. (1980). Aid, savings, and growth revisited, Oxford Bulletin of Economics and Statistics, 79-96.

[29] Dambisa M. (2009a). L'aide fatale, édition Jean-Claude Lattès pour la traduction française.

[30] Moyo, D. (2009b). Why Foreign Aid Is Hurting Africa. The Wall Street Journal.

[31] Murhula, Kitsali, K. et Mushagalusa, A. (2020). Pauvreté et Secteur Informel à Bukavu: Profil des Détaillants des Produits Agricoles à

I'Ere de la Pandémie COVID-19, Journal of Economics, Finance and Accounting Studies, 1 (2), 01-10

[32] Balasha M,Lebon, H. ,Maurice, K. ., Benjamin,M. B., Faustin, B. M., \& Jean-Helene, K. K. (2020). Understanding the Roles of Street Vendors of Agricultural Commodities duringthe COVID-19 Outbreak in the Informal Economy. Open Journal of Social Sciences, 8, 115-129. https://doi.org/10.4236/jss.2020.89008

[33] Nurske, R. (1953). Problems of capital formation in underdeveloped contries, Oxford University Press.

[34] Papenek, F. (1973). Aid, Foreign Private Investment, savings, and Growth in Less Developed Countries, Journal of Political Economy, $120-130$

[34] Perkins, D., Radelet, S. et Lindauer D. (2014). Economie du développement, Editions Nouveaux Horizons.

[35] PNUD (2010). Eliminer la pauvreté en 2015 : objectif du Millénaire pour le Développement. Rapport de 2010, https:// undp.org. [36] PNUD. (2017). Croissance inclusive, développement durable et défi de la décentralisation en République démocratique du Congo, Rapport national sur le développement humain 2016.

[37] PNUD (2019). Les inégalités de développement humain au XXle siècle : Note d'information à l'intention des pays concernant le Rapport sur le développement humain, $h t t p: / / h d r$.undp.org/sites/all/themes/hdr_theme/country-notes/fr/COD.pdf.

[38] Radelet, S., Clemens, M. et Bhavnani, R. (2004). Aide et croissance : débat encours et données nouvelles, Center for Global Development.

[39] Rosenstein, R., (1943). Problems of Industrialisation of Eastern and South-Eastern Europe, The Economic Journal, 202-211, https://doi.org/10.2307/2226317

[40] Rostow, W. (1962). Les étapes de la croissance économique, traduit de l’américain par M.-J. Du Rouret, paris, Editions du seuil, 200p. [41] Sachs, J. (2005). The end of poverty: economic possibilities for our time. New York: The Penguin Press.

[42] Stiglitz, J. (2002). Overseas Aid is Money Well Spent, Financial Times.

[43] Stiglitz, J. (2012). Le prix de l'inégalité, W.W Norton, New York. Traduit de l'américain par Françoise et Chemla.

[44] Tang, K. et Bundhoo D. (2017). Foreign Aid and Economic Growth in Developing Countries: Evidence from Sub-Saharan Africa, Theoretical Economics Letters, 1473-1491, http://www.scirp.org/journal/tel.

[45] Tshimanga, C. (2012). Évolution de la pauvreté en République Démocratique du Congo,

https://www.researchgate.net/publication/319468687. Consulté le 22 aout 2020. 